\title{
A FUNDAMENTAL SYSTEM OF INVARIANTS OF A MODULAR GROUP OF TRANSFORMATIONS *
}

\author{
BY \\ JOHN SIDNEY TURNER
}

1. Introduction. Let $G$ be any given group of $g$ homogeneous linear transformations on the indeterminates $x_{1}, \cdots, x_{n}$, with integral coefficients taken modulo $m$. Hurwitz $\dagger$ raised the question of the existence of a finite fundamental system of invariants of $G$ in the case where $m$ is a prime $p$, and obtained an affirmative answer when $g$ is prime to $p$. Dickson $\ddagger$ subsequently obtained an affirmative answer for any $g$.

The general case presents great difficulty, owing to the fact that resolution into irreducible factors with respect to a composite modulus is not, in general, unique. The present investigation is confined to the case in which there are two indeterminates $x, y$, and $m$ is the square of a prime $p$. The given group will be denoted by $H$, the notation $G$ being retained when $m=p$. It is proved that the $p^{2}+1$ invariants

$$
L^{p}, Q^{p}, p L^{\alpha} Q^{\beta} \quad(\alpha, \beta=0,1, \cdots, p-1 ; \alpha, \beta \text { not both zero }),
$$

where

$$
L=y x^{p}-x y^{p}, \quad Q=\left(x^{p^{2}-1}-y^{p^{2}-1}\right) /\left(x^{p-1}-y^{p-1}\right),
$$

form a fundamental system of (independent) invariants of the group $H$.

2. Consider the group $H$ of all linear homogeneous transformations modulo $p^{\text {: }}$ :

$$
x^{\prime} \equiv a x+b y, \quad y^{\prime} \equiv c x+d y, \quad a d-b c \equiv 1 \quad\left(\bmod p^{2}\right),
$$

where $a, b, c, d$ are integers. To each transformation of $H$ corresponds a unique transformation of the group $G$ :

(2) $\quad x^{\prime} \equiv a_{1} x+b_{1} y, \quad y^{\prime} \equiv c_{1} x+d_{1} y, \quad a_{1} d_{1}-b_{1} c_{1} \equiv 1 \quad(\bmod p)$, where $a_{1}, b_{1}, c_{1}, d_{1}$ are integers. In fact, we have only to choose

$$
a_{1} \equiv a, \quad \cdots, \quad d_{1} \equiv d \quad(\bmod p) .
$$

Conversely; to each transformation (2) corresponds one or more transformations (1). For, we can choose $a \equiv a_{1}, \cdots, d \equiv d_{1}(\bmod p)$ so that

* Presented to the Society, April 15, 1922.

†Archiv der Mathematik und Physik (3), vol. 5 (1903), p. 25.

$\ddagger$ The Madison Colloquium, Lect. III. 
$a d-b c \equiv 1\left(\bmod p^{2}\right)$. For example, if $a_{1} \neq 0(\bmod p)$ we may take $a \equiv a_{1}, b \equiv b_{1}, c \equiv c_{1}(\bmod p)$, and determine $d$ by $a d-b c \equiv 1\left(\bmod p^{2}\right)$; evidently $d \equiv d_{1}(\bmod p)$.

Hence if we reduce all the transformations of $H$ modulo $p$, we obtain all the transformations of $G$.

3. Definition. A rational and integral invariant of $H$ is a polynomial $I(x, y)$ in $x$ and $y$ with integral coefficients, which remains unchanged modulo $p^{2}$ under every transformation (1). That is,

$$
I\left(x^{\prime}, y^{\prime}\right) \equiv I(a x+b y, c x+d y) \equiv I(x, y)
$$

for all integers $a, \cdots, d$ such that $a d-b c \equiv 1\left(\bmod p^{2}\right)$.

Evidently any rational and integral invariant is a sum of homogeneous invariants; hence we restrict the investigation to the latter.

4. Theorem I. Let $I(x, y)$ be a rational and integral invariant of $H$, and let $I_{1}(x, y)$ be the polynomial obtained from $I(x, y)$ by replacing each coefficient by its positive or zero residue modulo $p$. Then $I_{1}(x, y)$ will be a rational and integral invariant of $G$.

We have (3) for all transformations of $H$. Now

$$
I(x, y) \equiv I_{1}(x, y)
$$

and

$$
\begin{aligned}
I(a x+b y, c x+d y) & \equiv I_{1}(a x+b y, c x+d y) \\
& \equiv I_{1}\left(a_{1} x+b_{1} y, c_{1} x+d_{1} y\right) \quad(\bmod p),
\end{aligned}
$$

hence

$$
I_{1}\left(x^{\prime}, y^{\prime}\right) \equiv I_{1}\left(a_{1} x+b_{1} y, c_{1} x+d_{1} y\right) \equiv I_{1}(x, y) \quad(\bmod p),
$$

and by $\S 2$ this is true for all transformations of $G$.

5. Now (Madison Colloquium, pp. 34-38),

$$
I_{1}(x, y) \equiv k T_{1}^{\alpha_{1}} T_{2}^{\alpha_{2}} \cdots T_{i}^{\alpha_{i}} \ldots T_{r}^{\alpha_{r} *}
$$

where $k$ is an integer,

$$
T_{1}=L, \quad T_{2}=Q, \quad T_{i}=R_{i}\left(L^{\lfloor p(p-1)}, \dagger Q^{\ddagger(p+1)}\right) \quad(i=3,4, \cdots, r),
$$

$R_{i}$ being a polynomial in its two arguments, with integral coefficients; moreover the $T_{i}(i=1,2, \cdots, r)$ contain no multiple factors, and are relatively prime modulo $p$. Hence

$$
I(x, y) \equiv k T_{1}^{\alpha_{1}} T_{2}^{\alpha_{2}} \cdots T_{i}^{\alpha_{i}} \ldots T_{r}^{\alpha_{r}}+p F(x, y)
$$

where $F(x, y)$ denotes a polynomial in $x, y$ with integral coefficients.

* In the discussion which follows, if any $\alpha_{i}$ is zero the corresponding $T_{i}$ is to be suppressed. † If $p=2$, we omit the divisor 2 in the exponents. 
6. Discussion of equation (5). Apply to $I(x, y)$ the transformation

$$
x^{\prime} \equiv x+p y, \quad y^{\prime} \equiv y \quad\left(\bmod p^{2}\right),
$$

expand by Taylor's Theorem, and denote the partial derivative of $T_{i}$ with respect to $x$ by $T_{i}^{\prime}$. Then

$$
\begin{aligned}
I(x+p y, y) & \equiv I(x, y)+p y k T_{1}^{\alpha_{1}-1} \cdots T_{i}^{\alpha_{i}-1} \\
& \times \cdots T_{r}^{\alpha_{r}-1} \sum_{i=1}^{r} \alpha_{i} T_{1} \cdots T_{i-1} T_{i}^{\prime} T_{i+1} \cdots T_{r}\left(\bmod p^{2}\right) .
\end{aligned}
$$

Since (6) is a transformation of $H$,

$$
I(x+p y, y) \equiv I(x, y) \quad\left(\bmod p^{2}\right) .
$$

Hence either $k \equiv 0(\bmod p)$, in which case the right member of $(5)$ reduces to its second term, or

$$
\sum_{i=1}^{r} \alpha_{i} T_{1} \cdots T_{i-1} T_{i}^{\prime} T_{i+1} \cdots T_{r} \equiv 0 \quad(\bmod p) .
$$

Let $\left(g_{i}, 1\right)$ be a point at which $T_{i}(x, y)$ vanishes. Then, for $j \neq i, T_{j}(x, y)$ cannot vanish at $\left(g_{i}, 1\right)$; for, in that event, $T_{j}(x, y)$ would be a factor of $T_{i}(x, y)$ modulo $p,{ }^{*}$ contrary to $\S 5$. Therefore from (8) we have

$$
\alpha_{i} T_{i}^{\prime}\left(g_{i}, 1\right) \equiv 0
$$

$(\bmod p)$.

Hence either $\alpha_{i} \equiv 0$, or $T_{i}^{\prime}\left(g_{i}, 1\right) \equiv 0(\bmod p)$. In the latter case, by a known theorem on Galois imaginaries, $T_{i}(x, 1)$ and $T_{i}^{\prime}(x, 1)$ have a common factor with integral coefficients modulo $p$. But $(\S 5) T_{i}(x, 1)$ contains no multiple factor modulo $p$. Therefore $T_{i}^{\prime}(x, 1) \equiv 0(\bmod p)$, whence

$$
T_{i}^{\prime}(x, y) \equiv 0 \quad(\bmod p) .
$$

Hence we have

TheOREM II. In equation (5), for each $i=1, \cdots, r$, either $\alpha_{i}$ is a multiple of $p$, or $T_{i}^{\prime}(x, y) \equiv 0(\bmod p)$.

Corollary $1 . \alpha_{1} \equiv 0(\bmod p)$.

For $T_{1}=y x^{p}-x y^{p}$; hence $T_{1}^{\prime}=p y x^{p-1}-y^{p} \equiv \equiv 0(\bmod p)$.

Corollary 2. $\alpha_{2} \equiv 0(\bmod p)$.

For

$$
T_{2}=x^{p(p-1)}+x^{(p-1)^{2}} y^{p-1}+\cdots+x^{p-1} y^{(p-1)^{2}}+y^{p(p-1)},
$$

hence $T_{2}^{\prime} \equiv 0(\bmod p)$.

Corollary 3. If $\alpha_{i}=p \beta_{i}$ for $i>2$,

$$
T_{i}^{\alpha_{i}} \equiv S_{i}\left(L^{p}, Q^{p}\right) \quad(\bmod p),
$$

where $S_{i}$ is a polynomial in its arguments, with integral coefficients.

* The Madison Colloquium, p. 38.

† Dickson, Lecture Notes on Double Modulus and Galois Imaginaries, §5. 
For if we expand

$$
T_{i}^{\alpha_{i}}=\left[R_{i}\left(L^{\frac{1}{2} p(p-1)}, Q^{\frac{1}{3}(p+1)}\right)\right]^{p \beta_{i}},
$$

we observe that in each term the exponent of $L$ is a multiple of $p$ and that either the exponent of $Q$ or the coefficient of the term is a multiple of $p$.

7. Discussion of $T_{i}^{\prime}(x, y) \equiv 0(\bmod p)$. Write

$$
T_{i}(x, y)=\sum_{r=0}^{n} A_{r} l^{n-r} q^{r}
$$

where $l=L^{\frac{1}{p} p(p-1)},^{*} q=Q^{\frac{1}{2}(p+1)}$, and the coefficients $A_{r}$ are integers; then

$$
T_{i}^{\prime}(x, y)=\sum_{r=0}^{n-1} A_{r}(n-r) l^{n-r-1} l^{\prime} q^{r}+\sum_{r=1}^{n} A_{r} r l^{n-r} q^{r-1} q^{\prime} \equiv 0 \quad(\bmod p),
$$

where $l^{\prime}, q^{\prime}$ denote the partial derivatives of $l, q$ with respect to $x$. Evidently $l^{\prime} \equiv 0, q^{\prime} \equiv 0(\bmod p)$; therefore

$$
\sum_{r=1}^{n} r A_{r} l^{n-r} q^{r-1} \equiv 0 \quad(\bmod p)
$$

Each term of (13) is the product of the preceding by $c q / l, c$ a constant; the degree in $x$ of $q / l$ is $\frac{1}{2}\left(p^{2}-p\right)$, $\dagger$ hence the degrees in $x$ of the successive terms increase by $\frac{1}{2}\left(p^{2}-p\right)$. Equating coefficients of $x$, we find in succession

$$
n A_{n} \equiv 0, \quad \cdots, \quad r A_{r} \equiv 0, \quad \cdots, \quad A_{1} \equiv 0 \quad(\bmod p) .
$$

Hence in each term of $\sum_{r=1}^{n} A_{r} l^{n-r} q^{r}$, either the coefficient $A_{r}$ or the exponent of $q$ is a multiple of $p$, and we have

Theorem III. If $T_{i}^{\prime}(x, y) \equiv 0(\bmod p)$, then

$$
T_{i}(x, y) \equiv S_{i}\left(L^{p}, Q^{p}\right)
$$

$(\bmod p)$,

where $S_{i}$ denotes a rational and integral function of its arguments, with integral coefficients.

CoRollary. $\quad\left[T_{i}(x, y)\right]^{\alpha_{i}}$ is a polynomial in $L^{p}, Q^{p}$, with integral coefficients, modulo $p$.

8. Theorem IV. $L^{p}$ is invariant under the group $H$.

Write $L\left(x^{\prime}, y^{\prime}\right)=e, L(x, y)=f$, where $x^{\prime}, y^{\prime}$ are derived from $x, y$ by any transformation $(2)$ of the group $G$; then $\ddagger e-f \equiv 0(\bmod p)$. Hence $e-f \equiv 0(\bmod p)$ for every transformation $(1)$ of $H$. For if, as in $\S 2$, we choose $a_{1} \equiv a, \cdots, d_{1} \equiv d$ modulo $p$, we have

$L(a x+b y, c x+d y) \equiv L\left(a_{1} x+b_{1} y, c_{1} x+d_{1} y\right) \equiv L(x, y) \quad(\bmod p)$.

* If $p=2$, we omit the divisor 2 in the exponents.

$\dagger$ If $p=2$, the degree is 2 .

$\ddagger$ The Madison Colloquium, p. 35. 
Also

$$
\begin{aligned}
e^{p}-f^{p}= & (e-f+f)^{p}-f^{p} \\
& =(e-f)\left[(e-f)^{p-1}+\cdots+\frac{1}{2} p(p-1)(e-f) f^{p-2}+p f^{p-1}\right],
\end{aligned}
$$

and each factor on the right is identically congruent to zero modulo $p$; hence $e^{p}-f^{p} \equiv 0\left(\bmod p^{2}\right)$; that is

$$
[L(a x+b y, c x+d y)]^{p} \equiv[L(x, y)]^{p} \quad\left(\bmod p^{2}\right)
$$

for every transformation of $H$.

CoRollary 1. In the same way, it can be proved that $Q^{p}$ is invariant under the group $H$.

CoRollary 2. $p L^{\alpha} Q^{\beta}$ is invariant under the group $H$.

9. Theorem V. Any rational and integral invariant of the group $H$ is a rational and integral function, with integral coefficients, of the $p^{2}+1$ invariants $L^{p}, Q^{p}, p L^{\alpha} Q^{\beta}(\alpha, \beta=0,1, \cdots, p-1 ; \alpha, \beta$ not both zero $)$. Conversely, any such function is an invariant of $H$.

In (5), the term $k T_{1}^{\alpha_{1}} T_{2}^{\alpha_{2}} \ldots T_{r}^{\alpha_{r}}$ is an invariant of $H$. For if any $\alpha_{i} \equiv 0$ $(\bmod p)$, then by Theorems II and IV with their corollaries, $T_{i}^{\alpha_{i}}$ is an invariant; $T_{1}=L, T_{2}=Q, \alpha_{1} \equiv \alpha_{2} \equiv 0(\bmod p)$. While if $\alpha_{j} \neq 0(\bmod p)$, $T_{j}^{\prime} \equiv 0$, and by Theorems III and IV with their corollaries $T_{j}^{\alpha_{j}}$ is an invariant.

Hence the second term $p F(x, y)$ of (5) is an invariant of $H$, and it follows from $\S 2$ that $F(x, y)$ is an invariant of $G$. Therefore $p F(x, y)$ is the product of $p$ by a polynomial in $L$ and $Q$. It follows that if $I(x, y)$ is any rational and integral invariant of $H$,

$$
I(x, y) \equiv S\left(L^{p}, Q^{p}, p L^{\alpha} Q^{\beta}\right) \quad\left(\bmod p^{2}\right),
$$

where $p L^{\alpha} Q^{\beta}$ denotes the set $p L, p Q, p L^{2}, p L Q, \cdots, p L^{p-1} Q^{p-1}$, and $S$ denotes a rational and integral function of its arguments, with integral coefficients.

Conversely, any rational and integral function of $L^{p}, Q^{p}, p L^{\alpha} Q^{\beta}$, with integral coefficients, is a sum of invariants, and is therefore itself an invariant. Hence these $p^{2}+1$ invariants form a fundamental system.

10. ThEOREM VI. The invariants of the fundamental system are independent. In view of the coefficients $p$, neither $L^{p}$ nor $Q^{p}$ can be expressed as a polynomial in the remaining invariants, with integral coefficients. Assume that $p L^{\alpha_{1}} Q^{\beta_{1}}, \alpha_{1} \leqq p-1, \beta_{1} \leqq p-1$, can be so expressed. Then

$$
p L^{\alpha_{1}} Q^{\beta_{1}} \equiv P\left(L^{p}, Q^{p}, p L^{\alpha} Q^{\beta}\right)
$$

identically in $x, y$. We may suppose that $P$ contains no group of terms which vanishes identically modulo $p^{2}$. Let $m L^{\alpha_{2}} Q^{\beta_{2}}$ be any term of $P$; then $p L^{\alpha_{1}} Q^{\beta_{1}}$ and $m L^{\alpha_{2}} Q^{\beta_{2}}$ must be of the same total degree in $x, y$, and also of the same 
degree in $x$ alone. Therefore

$$
\begin{aligned}
\alpha_{1}(p+1)+\beta_{1} p(p-1) & =\alpha_{2}(p+1)+\beta_{2} p(p-1), \\
\alpha_{1} p+\beta_{1} p(p-1) & =\alpha_{2} p+\beta_{2} p(p-1),
\end{aligned}
$$

whence $\alpha_{1}=\alpha_{2}, \beta_{1}=\beta_{2}$. Hence $P$ consists of the single term $p L^{\alpha_{1}} Q^{\beta_{1}}$. Evidently $p L^{\alpha_{1}} Q^{\beta_{1}}$ is not a product of fundamental invariants, hence the theorem is proved.

11. If we consider the total group

$$
\begin{array}{cc}
x^{\prime} \equiv a x+b y, \quad y^{\prime} \equiv c x+d y & \left(\bmod p^{2}\right), \\
a d-b c \neq 0 & (\bmod p),
\end{array}
$$

we find, exactly as in Theorem IV, that $Q^{p}$ is an absolute invariant, and that $L^{p}, p L^{\alpha} Q^{\beta}$ are relative invariants of indices $p, \alpha$, respectively.

Iowa State College,

AMEs, Iowa. 